\title{
MicroRNA-27a-3p promotes proliferation and migration by up-regulation of perlecan in murine fibroblast cell line $\mathrm{NIH} / 3 \mathrm{~T} 3$
}

\section{Type}

Research paper

\section{Keywords}

SCAR, microRNA-27a-3p, proliferation, migration, perlecan, MAPK/ERK/PI3K/AKT

\begin{abstract}
Introduction

Skin scar is a common cutaneous complication, the outcome of which is unpleasant. Several microRNAs (miRs) participate in the process of skin scar formation. We aimed to explore the role of miR-27a-3p in NIH/3T3 mouse fibroblasts as well as the downstream protein and signaling cascades.

Material and methods

miR-27a-3p was aberrantly expressed in NIH/3T3 cells, followed by measurements of cell viability, migration and expressions of proteins related to proliferation and migration. Perlecan expression in cells aberrantly expressing miR-27a-3p was examined by Western blot analysis. Reporter gene assay was conducted to assess the relationship between miR-27a-3p and perlecan. Then, whether miR-27a-3p affected NIH/3T3 cells through regulating perlecan was ascertained. The effects of aberrantly expressed miR-27a-3p and perlecan on expression levels of VEGF, bFGF and key kinases in the MAPK/ERK and the PI3K/AKT pathways were detected.

\section{Results}

Cell viability and migration were enhanced and protein expression levels of Cyclin D1, MMP-2 and MMP-9 were up-regulated by miR-27a-3p overexpression in NIH/3T3 cells. Then, we found that perlecan was positively correlated with miR-27a-3p expression, and its knockdown abrogated the effects of miR-27a-3p overexpression on NIH/3T3 cells. Finally, we found that the expression levels of VEGF and bFGF as well as phosphorylated levels of MAPK, ERK, PI3K and AKT were increased by miR-27a-3p overexpression, and those increases were reversed by perlecan knockdown.
\end{abstract}

\section{Conclusions}

miR-27a-3p promotes proliferation and migration of NIH/3T3 cells through up-regulating perlecan expression. Meanwhile, miR-27a-3p up-regulates expression levels of VEGF and bFGF, and activates MAPK/ERK and PI3K/AKT pathways through up-regulating perlecan expression. 


\title{
MicroRNA-27a-3p promotes proliferation and migration by up-regulation of perlecan in murine fibroblast cell line NIH/3T3
}

Running title: Role of miR-27a-3p in fibroblasts

\begin{abstract}
Background: Skin scar is a common cutaneous complication, the outcome of which is unpleasant. Several microRNAs (miRs) participate in the process of skin scar formation. We aimed to explore the role of miR-27a-3p in NIH/3T3 mouse fibroblasts as well as the downstream protein and signaling cascades.
\end{abstract}

Methods: miR-27a-3p was aberrantly expressed in NIH/3T3 cells, followed by measurements of cell viability, migration and expressions of proteins related to proliferation and migration. Perlecan expression in cells aberrantly expressing miR-27a-3p was examined by Western blot analysis. Reporter gene assay was conducted to assess the relationship between miR-27a-3p and perlecan. Then, whether miR-27a-3p affected NIH/3T3 cells through regulating perlecan was ascertained. The effects of aberrantly expressed miR-27a-3p and perlecan on expression levels of VEGF, bFGF and key kinases in the MAPK/ERK and the PI3K/AKT pathways were detected.

Results: Cell viability and migration were enhanced and protein expression levels of Cyclin D1, MMP-2 and MMP-9 were up-regulated by miR-27a-3p overexpression in NIH/3T3 cells. Then, we found that perlecan was positively correlated with miR-27a-3p expression, and its knockdown abrogated the effects of miR-27a-3p overexpression on NIH/3T3 cells. Finally, we found that the expression levels of VEGF and bFGF as well as phosphorylated levels of MAPK, 
ERK, PI3K and AKT were increased by miR-27a-3p overexpression, and those increases were reversed by perlecan knockdown.

Conclusion: miR-27a-3p promotes proliferation and migration of $\mathrm{NIH} / 3 \mathrm{~T} 3$ cells through up-regulating perlecan expression. Meanwhile, miR-27a-3p up-regulates expression levels of VEGF and bFGF, and activates MAPK/ERK and PI3K/AKT pathways through up-regulating perlecan expression.

Keywords: Scar; microRNA-27a-3p; proliferation; migration; perlecan; MAPK/ERK/PI3K/AKT 


\section{Introduction}

Skin scar is a common cutaneous complication that is caused by injuries to the skin due to burn, surgery, trauma or infection (Alkhalil et al. 2015). Unlike the normotrophic scars which are slightly broadened white lines, pathologic scarring are divided into atrophic, contracted, hypertrophic or keloid scars on the basis of the clinical behavior and appearance (Bayat et al. 2003; Bijlard et al. 2017). It has been estimated that approximately 100 million patients suffer from any form of pathologic scars in developed countries annually (Brown et al. 2008). Despite of the development of modern medicine, esthetic, psychological and physical complaints because of dermal scars contribute to decrease of life quality (Gras et al. 2015). The unpleasant outcomes of skin scars make the molecular mechanisms underlying scar formation become a hotspot recently.

Cutaneous wound repair is a well-orchestrated process and is divided into three overlapping phases which are inflammatory response, formation of the granulation tissues and remodeling of matrix (Reinke and Sorg 2012). Fibroblasts are the main contributing cells that participate in all the three phase. More importantly, fibroblasts are responsible for maintaining of the physical integrity in connective tissues by means of the synthesis and remodeling of the extracellular matrix (ECM) (Vedrenne et al. 2012). Hypertrophic and keloid scars, major forms of pathologic scars, are raised dermal scars, and the excessive extracellular matrix (ECM) synthesis, delayed apoptosis and elevated migration and proliferation are characters of fibroblasts in hypertrophic and keloid scars (Zhu et al. 2013; Suarez et al. 2015). In addition, fibroblast proliferation and migration have been reported to play pivotal roles in skin wound healing ( $\mathrm{He}$ et al. 2017). Therefore, the mechanisms underlying excessive proliferation and migration of fibroblasts are of great importance.

MicroRNAs (miRNAs/miRs), the small single stranded RNAs that cannot encode proteins, have been identified to participate in numerous biological processes through posttranscriptional modifications and/or modulation of translation processes (Yang et al. 2015; Krishnan et al. 2017; 
Sliwinska et al. 2017). Recently, more and more miRNAs are found to be involved in dermal scar formation. For example, miR-31-5p knockdown has been reported to effectively reduce hypertrophic scar formation in hypertrophic scar fibroblasts under hypoxia (Wang et al. 2017). The proliferation of human hypertrophic scar fibroblasts was also repressed by miR-98 via targeting Col1A1 (Bi et al. 2017). miR-27a-3p has widely reported to regulate cell proliferation and migration via acting as either a oncogene or a tumor suppressor gene (Li et al. 2017; Liang et al. 2017). However, the functional roles of miR-27a-3p in fibroblasts are far from understood. Perlecan, encoded by heparin sulfate proteoglycan 2 (HSPG2) gene, is named from the appearance of a "beads on a string" under a rotary shadow electron microscopy (Warren et al. 2015). Previous studies have reported that perlecan can be observed in the subendothelial extracellular matrix of the vascular wall and play critical roles in cell adhesion, migration and proliferation (Lord et al. 2014; Nakamura et al. 2015; Nonaka et al. 2015).

Herein, in this study, we firstly identified the regulatory roles of miR-27a-3p in proliferation and migration of mouse fibroblasts cell line, NIH/3T3, and then we explored the possible molecular mechanism related to perlecan, as well as downstream signaling cascades.

\section{Materials and methods}

\section{Cell culture}

NIH/3T3 mouse fibroblasts (ATCC ${ }^{\circledR}$ CRL-1658 ${ }^{\mathrm{TM}}$ ) were obtained from American Type Culture Collection (ATCC; Manassas, VA, USA). NIH/3T3 cells were grown in Dulbecco's modified Eagle's medium (DMEM; Thermo Fisher Scientific, Waltham, MA, USA) containing $10 \%$ fetal bovine serum (FBS; Thermo Fisher Scientific) at $37^{\circ} \mathrm{C}$ in a humidified atmosphere with $5 \%$ $\mathrm{CO}_{2}$.

\section{Cell transfection}


miR-27a-3p mimic, miR-27a-3p inhibitor, their negative control (miR-NC), small interfering RNA targeting perlecan (si-perlecan) and its negative control (si-NC) were synthesized by GenePharma Company (Shanghai, China). miRNAs or si-perlecan were transfected into NIH/3T3 cells with the help of lipofectamine 3000 reagent (Invitrogen, Carlsbad, CA, USA), according to the supplier's instructions.

\section{Cell viability assay}

Cell viability of $\mathrm{NIH} / 3 \mathrm{~T} 3$ cells after aberrant expression of miR-27a-3p and perlecan was assessed by Cell Counting Kit-8 (CCK-8, Boster Biological Technology Ltd., Wuhan, China). In brief, after cell transfection, $5 \times 10^{3}$ cells were seeded in each well of 96-well plates and incubated for $24 \mathrm{hr}\left(37^{\circ} \mathrm{C}, 5 \% \mathrm{CO}_{2}\right)$. Then, CCK-8 solution $(10 \mu \mathrm{L})$ was added into each well, and the cells were incubated for additional $1 \mathrm{hr}\left(37^{\circ} \mathrm{C}, 5 \% \mathrm{CO}_{2}\right)$. A Microplate Reader (Bio-Rad, Hercules, CA, USA) was utilized for measurements of absorbance at $450 \mathrm{~nm}$.

\section{Tranwell assay}

Cell migration of NIH/3T3 cells after aberrant expression of miR-27a-3p and perlecan was examined using the modified 24-well Boyden chambers $(8 \mu \mathrm{m}$ pore size, Corning Incorporated, Corning, NY, USA). In brief, after cell transfection, cells suspended in $200 \mu \mathrm{L}$ DMEM were seeded on the upper compartments, whereas $600 \mu \mathrm{L}$ DMEM containing 10\% FBS was added into the lower compartments. Cells were subjected to a humidified incubator with $5 \% \mathrm{CO}_{2}$ at $37^{\circ} \mathrm{C}$ for $24 \mathrm{hr}$, followed by fixation with methanol. After that, non-traversed cells on the upper surface of the filter were carefully removed with a cotton swab. The traversed cells which stayed on the lower side of the filter were stained with $1 \%$ crystal violet solution and counted under a microscopy (Olympus, Tokyo, Japan). 


\section{Stem-loop RT-PCR based quantification of miR-27a-3p}

After cell transfection, total RNAs were extracted from NIH/3T3 cells using TRIzol reagent (Invitrogen) and DNase I (Ambion, Norwalk, CT, USA). The isolated RNAs were quantified by NanoDrop 2000 (Thermo Fisher Scientific), and 500 ng RNAs were converted to miRNA specific cDNA utilizing the Taqman MicroRNA Reverse Transcription Kit (Applied Biosystems, Foster City, CA, USA). The program was as follows: $16^{\circ} \mathrm{C}$ for $30 \mathrm{~min}, 42^{\circ} \mathrm{C}$ for $30 \mathrm{~min}$ and $85^{\circ} \mathrm{C}$ for $5 \mathrm{~min}$. The real-time PCR was performed with the Taqman Universal Master Mix II without UNG (Applied Biosystems), as suggested by the manufacturer. The program was as follows: $95^{\circ} \mathrm{C}$ for $10 \mathrm{~min}$, and 40 cycles of $95^{\circ} \mathrm{C}$ for $15 \mathrm{~s}$ and $60^{\circ} \mathrm{C}$ for $1 \mathrm{~min}$. Expression levels of miR-27a-3p were analyzed using $2^{-\Delta \Delta C t}$ method (Livak and Schmittgen 2001), normalizing to U6.

\section{Reporter gene assay}

The pGL3-perlecan vector was prepared as previously descried (Zhang et al. 2010). For reporter gene assay, $0.4 \mu \mathrm{g}$ of reporter gene construct was transfected into NIH/3T3 cells using lipofectamine 3000 reagent in line with the manufacturer's instruction. This transfection was done concurrently with the transfection of miR-27a-3p inhibitor. The relative luciferase activity was detected using dual luciferase reporter assay (Promega, Madison, WI, USA).

\section{Western blot analysis}

After cell transfection, NIH/3T3 cells were lysed in RIPA buffer (Beyotime, Shanghai, China) containing $1 \mathrm{mM}$ PMSF (Beyotime). Cell lysates were centrifuged at $14000 \mathrm{~g}$ for $15 \mathrm{~min}$ at $4^{\circ} \mathrm{C}$, and the supernatants were collected for quantification. Then, supernatants containing proteins $(\sim 30 \mu \mathrm{g})$ were separated by SDS-PAGE and transferred onto polyvinylidene difluoride (PVDF) 
membranes. After blockage with 5\% non-fat milk, PVDF membranes were incubated with primary antibody at $4^{\circ} \mathrm{C}$ overnight. The primary antibodies were anti-cyclinD1 (ab134175), anti-matrix $\quad$ metalloproteinase $\quad(M M P)-2 \quad(a b 97779), \quad$ anti-MMP-9 $\quad($ ab58803), anti-mitogen-activated protein kinase (MAPK; ab197348), anti-phospho (p)-MAPK (ab47363), anti-extracellular signal-regulated kinase (ERK; ab115799), anti-p-ERK (ab214036), anti-phosphatidylinositol-3-kinase (PI3K; ab191606), anti-p-PI3K (ab182651), anti- $\beta$-actin (ab8229, all Abcam), anti-AKT (9272), anti-p-AKT (9271, both Cell Signaling Technology, Beverly, MA, USA), anti-vascular endothelial growth factor (VEGF; sc-7269), anti-basic fibroblast growth factor (bFGF; sc-271847) and anti-perlecan (sc-33707, all Santa Cruz, Santa Cruz, CA, USA) antibodies. After rinsing thrice, PVDF membranes were reacted with HRP-conjugated secondary antibodies (Abcam) at room temperature for $1 \mathrm{hr}$. After rinsing again, proteins in the PVDF membranes were visualized using an ECL Western blotting detection reagent (GE Healthcare, Braunschweig, Germany). Relative intensity of the protein bands was analyzed using ImageJ 1.47 software (National Institutes of Health, Bethesda, MD, USA).

\section{Statistical analysis}

Results were presented as the mean \pm standard deviation (SD) of at least three independent experiments. Statistically significant differences between groups were determined by Graphpad Prism 5 software (GraphPad, San Diego, CA, USA). The $P$-values were calculated using unpaired two-tailed $t$-test or multiple $t$-tests. A $P<0.05$ was considered to indicate a statistically significant difference.

\section{Results}

miR-27a-3p promotes proliferation and migration of $\mathrm{NIH} / 3 \mathrm{T3}$ cells 
miR-27a-3p mimic or miR-27a-3p inhibitor was transfected into NIH/3T3 cells to aberrantly express miR-27a-3p. Compared with the miR-NC group, miR-27a-3p levels were significantly increased by transfection with miR-27a-3p mimic and decreased by transfection with miR-27a-3p inhibitor (both $P<0.01$, Figure 1A). Results indicated that miR-27a-3p expression was successfully altered. Then, cell viability, migration and expression of proteins associated with proliferation and migration were all measured after aberrant expression of miR-27a-3p. Cell viability (Figure 1B) and migration (Figure 1D) were significantly enhanced by miR-27a-3p overexpression while reduced by miR-27a-3p inhibition relative to the miR-NC group (all $P<0.05$ ). In Figure 1C and 1E, protein expression levels of Cyclin D1, MMP-2 and MMP-9 were all significantly up-regulated by miR-27a-3p overexpression $(P<0.01$ or $P<$ $0.001)$ while down-regulated by miR-27a-3p inhibition $(P<0.05$ or $P<0.01)$ as compared to the miR-NC group. Those results collectively demonstrated that miR-27a-3p could promote $\mathrm{NIH} / 3 \mathrm{~T} 3$ cell proliferation and migration.

miR-27a-3p up-regulates perlecan expression in NIH/3T3 cells

Next, Western blot analysis and reporter gene assay were performed to explore the interaction between miR-27a-3p and perlecan. As evidenced in Figure 2A, miR-27a-3p overexpression remarkably up-regulated perlecan expression, and miR-27a-3p inhibition notably down-regulated perlecan expression, when compared to the miR-NC group (both $P<0.001$ ). Figure 2B showed that co-transfection with perlecan vector and increasing amounts of miR-27a-3p inhibitor led to significantly decrease in relative luciferase activity $(P<0.05, P<$ 0.01 or $P<0.001)$. These results indicated that miR-27a-3p positively regulated perlecan expression in NIH/3 3 cells might via binding to the promoter of perlecan. 
Subsequent experiments were carried out to ascertain whether miR-27a-3p affect NIH/3T3 cells via regulating perlecan expression. First of all, the knockdown efficiency of si-perlecan was confirmed by Western blot analysis. In Figure 3A, perlecan expression was significantly down-regulated by transfection with si-perlecan relative to the si-NC group $(P<0.001)$. Figure 3B and 3D showed that si-perlecan+miR-NC co-transfection notably reduced the viability and migration of $\mathrm{NIH} / 3 \mathrm{~T} 3$ cells, relative to miR-NC+si-NC co-transfection $(P<0.05)$. Moreover, compared to miR-27a-3p mimic + si-NC co-transfection group, the viability and migration of NIH/3T3 cells were both increased in miR-27a-3p mimic + si-perlecan co-transfection group $(P$ $<0.05$ or $P<0.01$ ). Similar results were found in Figure $3 \mathrm{C}$ and $3 \mathrm{E}$, which displayed that si-perlecan+miR-NC co-transfection noticeably down-regulated the protein expression levels of Cyclin D1, MMP-2 and MMP-9 (Figure 3E) in NIH/3T3 cells, compared to miR-NC+si-NC co-transfection $(P<0.01)$. Relative to miR-27a-3p mimic + si-NC co-transfection group, the protein expression levels of Cyclin D1, MMP-2 and MMP-9 were all up-regulated in miR-27a-3p mimic + si-perlecan co-transfection group mimic + si-NC $(P<0.01)$. Taken together, those results collectively proved that miR-27a-3p positively regulated perlecan expression might via binding to the promoter of perlecan. These findings also indicated that Overexpression of miR-27a-3p-induced up-regulation of perlecan expression might be a reason for the enhancements of proliferation and migration in NIH/3T3 cells.

miR-27a-3p up-regulates expression levels of VEGF and bFGF via up-regulating perlecan in NIH/3T3 cells

Effects of miR-27a-3p and perlecan on expression levels of VEGF and bFGF in NIH/3T3 cells were explored. As evidenced in Figure 4, the expression levels of VEGF and bFGF were dramatically elevated by miR-27a-3p overexpression and reduced by perlecan knockdown, as compared to the miR-NC+si-NC group $(P<0.01$ or $P<0.001)$. In addition, miR-27a-3p 
overexpression-induced up-regulations of VEGF and bFGF expression levels were notably abrogated by perlecan knockdown relative to the miR-27a-3p mimic + si-NC group $(P<0.01$ or $P<0.001)$. Data talked above suggested that miR-27a-3p could up-regulated expression levels of VEGF and bFGF via up-regulating perlecan expression in NIH/3T3 cells.

miR-27a-3p activates MAPK/ERK and PI3K/AKT pathways via up-regulating perlecan in NIH/3T3 cells

The involvements of signaling cascades in the miR-27a-3p-associated regulation were finally studied. In Figure 5A-B, phosphorylated levels of MAPK, ERK, PI3K and AKT were prominently increased by miR-27a-3p overexpression and decreased by perlecan knockdown, relative to the miR-NC+si-NC group (all $P<0.001$ ). More importantly, miR-27a-3p overexpression-induced up-regulations of MAPK, ERK, PI3K and AKT phosphorylated levels were significantly reversed by perlecan knockdown relative to the miR-27a-3p mimic + si-NC group (all $P<0.001$ ). These results proposed that miR-27a-3p overexpression could activate MAPK/ERK and PI3K/AKT pathways in NIH/3T3 cells, partially due to the up-regulation of perlecan.

\section{Discussion}

Considering that the current therapeutic measures for pathological scars have their limitations, it is urgently needed to explore innovative strategies for improving the outcome of skin scar formation. In our study, we interestingly found that miR-27a-3p overexpression could promote proliferation and migration of NIH/3T3 cells. Then, we revealed that the perlecan expression was positively correlated with the miR-27a-3p expression, and effects of miR-27a-3p overexpression on proliferation and migration of $\mathrm{NIH} / 3 \mathrm{~T} 3$ cells were reversed by perlecan knockdown. In addition, the protein expression levels of VEGF and bFGF as well as the protein 
phosphorylated levels of MAPK, ERK, PI3K and AKT were all increased by miR-27a-3p through up-regulating perlecan.

The functional roles of miR-27a, located on chromosome 19 (19p13.1), have been well studied in cancer cells. miR-27a-3p has been illustrated to act as an oncogene in several cancer types. For example, miR-27a-3p has been demonstrated to promote proliferation of glioma cells (Xu et al. 2013) and gastric carcinoma cells (Zhou et al. 2016). Both proliferation and migration were promoted by miR-27a-3p in renal cell carcinoma cells (Peng et al. 2015) and nasopharyngeal carcinoma cells ( $\mathrm{Li}$ and Luo 2017). Conversely, miR-27a-3p can inhibit proliferation and migration in esophageal squamous cell carcinoma (Zhu et al. 2014) and colorectal carcinoma (Bao et al. 2014) cells thereby acting as a tumor suppressor. Hence, it is curious that whether miR-27a-3p can affect proliferation and migration of fibroblasts. Results in our study showed that the viability and migration of $\mathrm{NIH} / 3 \mathrm{~T} 3$ cells were enhanced by miR-27a-3p overexpression while were reduced by miR-27a-3p inhibition. Cyclin D1 is a critical protein involved in regulation of cell proliferation. It can activates cyclin-dependent kinase (CDK) 4 and 6, thereby induces phosphorylation of retinoblastoma protein and expression of proliferation associated proteins, resulting in elevated proliferation ( $\mathrm{Li}$ et al. 2007). MMPs, especially MMP-2 and MMP-9, can degrade several ECM components. MMP-2 which degrades type IV collagen substrates and MMP-9 which degrades gelation substrates are highly related with cell migration (Webb et al. 2017). In our study, the up-regulations of Cyclin D1, MMP-2 and MMP-9, which are induced by miR-27a-3p overexpression, further consolidated the pro-proliferative and pro-migratory capacities of miR-27a-3p in fibroblasts.

As a key regulatory molecule in cell adhesion, migration and proliferation, perlecan has been demonstrated to participate in the process of skin scar formation (Gustafsson et al. 2013). Dos et al. reported that perlecan influenced the keratin 15-positive cell population fate in the epidermis of aging skin (Dos Santos et al. 2016). Therefore, in the current research, we next focused on the interaction between miR-27a-3p and perlecan to explore the regulatory mechanism of miR-27a-3p in fibroblasts. We found that miR-27a-3p could positively regulate the expression 
of perlecan in NIH/3T3 cells via binding to the promoter of perlecan. Perlecan knockdown inhibited the proliferation and migration of NIH/3T3 cells. Additionally, we revealed that the miR-27a-3p overexpression-induced increases of proliferation and migration of NIH/3T3 cells were abrogated by perlecan knockdown. Therefore, we concluded that up-regulation of perlecan might be a possible explanation for the effects of miR-27a-3p on fibroblasts.

VEGF is a glycoprotein that plays a critical role in angiogenesis. Previous studies have proved that VEGF expression is up-regulated in pathologic scars relative to the normal skins (Bock et al. 2006; Ong et al. 2007). bFGF is known as a mitogen and chemoattractant that can stimulate angiogenesis in fibroblasts (Ohno et al. 2016). bFGF is also proposed to promote proliferation, migration and differentiation of basic fibroblasts (Niu et al. 2015). Therefore, we next measured the alteration of VEGF and bFGF expression after aberrant expression of miR-27a-3p and perlecan. Results showed miR-27a-3p up-regulated protein expression levels of VEGF and bFGF, and these up-regulations were abrogated by perlecan knockdown. A previous study has proved that perlecan can act as a co-activator of VEGF and bFGF and thereby promote the binding of these two factors to their cognate receptors (Ishijima et al. 2012). Our results as well as the previous literature collectively proved that miR-27a-3p could up-regulated expression of VEGF and bFGF in fibroblasts through up-regulating perlecan expression.

The MAPK/ERK and PI3K/AKT pathways are important signal transduction pathways that participate in cell proliferation and migration (Sun et al. 2015; Chiappini et al. 2017; Wong et al. 2018). Zhou et al. have proved that the MAPK/ERK pathway is activated in gastric cancer cells overexpressing miR-27a-3p. Also in gastric cancer cells, Ding et al. have reported that miR-27a-3p promotes malignant behavior by activating the PI3K/AKT pathway (Ding et al. 2017). Therefore, we finally testified the involvements of these two pathways in NIH/3T3 cells. Results illustrated that miR-27a-3p could activate MAPK/ERK and PI3K/AKT pathways through up-regulating perlecan.

\section{Conclusions}


To summarize, we reported for the first time that miR-27a-3p could promote proliferation and migration of fibroblasts (NIH/3T3 cells). Perlecan was the downstream protein of miR-27a-3p. Moreover, miR-27a-3p up-regulated expression of VEGF and bFGF and activated the MAPK/ERK and PI3K/AKT pathways through up-regulating perlecan. Our study not only provided the regulatory mechanism of miR-27a-3p in fibroblasts, but also verified that both miR-27a-3p and perlecan might be therapeutic targets for pathological scar formation.

\section{Acknowledgements}

This research received no specific grant from any funding agency in the public, commercial or not-for-profit sectors.

\section{Conflict of interests}

The authors declare that they have no competing interests. 


\section{Reference}

Alkhalil A et al. (2015) A Translational Animal Model for Scar Compression Therapy Using an Automated Pressure Delivery System. Eplasty 15:e29

Bao $Y$ et al. (2014) Tumor suppressor microRNA-27a in colorectal carcinogenesis and progression by targeting SGPP1 and Smad2. PLoS One 9:e105991 doi: 10.1371/journal.pone.0105991

Bayat A, McGrouther DA, Ferguson MW (2003) Skin scarring. Bmj 326:88-92

Bi S, Chai L, Yuan X, Cao C, Li S (2017) MicroRNA-98 inhibits the cell proliferation of human hypertrophic scar fibroblasts via targeting Col1A1. Biol Res 50:22 doi: 10.1186/s40659-017-0127-6

Bijlard E, Uiterwaal L, Kouwenberg CA, Mureau MA, Hovius SE, Huygen FJ (2017) A Systematic Review on the Prevalence, Etiology, and Pathophysiology of Intrinsic Pain in Dermal Scar Tissue. Pain Physician 20:1-13

Bock O, Schmid-Ott G, Malewski P, Mrowietz U (2006) Quality of life of patients with keloid and hypertrophic scarring. Arch Dermatol Res 297:433-438 doi: 10.1007/s00403-006-0651-7

Brown BC, McKenna SP, Siddhi K, McGrouther DA, Bayat A (2008) The hidden cost of skin scars: quality of life after skin scarring. J Plast Reconstr Aesthet Surg 61:1049-1058 doi: 10.1016/j.bjps.2008.03.020

Chiappini PBO et al. (2017) Prognostic implications of phosphatidylinositol 3-kinase/AKT signaling pathway activation in gastric carcinomas. Arch Med Sci 13:1262-1268 doi: 10.5114/aoms.2016.60394

Ding L, Zhang S, Xu M, Zhang R, Sui P, Yang Q (2017) MicroRNA-27a contributes to the malignant behavior of gastric cancer cells by directly targeting $\mathrm{PH}$ domain and leucine-rich repeat protein phosphatase 2. J Exp Clin Cancer Res 36:45 doi: 10.1186/s13046-017-0516-2

Dos Santos M et al. (2016) Perlecan expression influences the keratin 15-positive cell population fate in the epidermis of aging skin. Aging (Albany NY) 8:751-768 doi: 10.18632/aging.100928

Gras C, Ratuszny D, Hadamitzky C, Zhang H, Blasczyk R, Figueiredo C (2015) miR-145 Contributes to Hypertrophic Scarring of the Skin by Inducing Myofibroblast Activity. Mol Med 21:296-304 doi: 10.2119/molmed.2014.00172

Gustafsson E, Almonte-Becerril M, Bloch W, Costell M (2013) Perlecan maintains microvessel integrity in vivo and modulates their formation in vitro. PLoS One 8:e53715 doi: 10.1371/journal.pone.0053715

He X, Dai J, Fan Y, Zhang C, Zhao X (2017) Regulation function of MMP-1 downregulated by siRNA on migration of heat-denatured dermal fibroblasts. Bioengineered 8:686-692 doi: 10.1080/21655979.2016.1267885

Ishijima M et al. (2012) Perlecan modulates VEGF signaling and is essential for vascularization in endochondral bone formation. Matrix Biology 31:234-245 doi: 10.1016/j.matbio.2012.02.006

Krishnan R, Nair AS, Dhar PK (2017) Computational study of 'HUB' microRNA in human cardiac diseases. Bioinformation 13:17-20 doi: 10.6026/97320630013017

Li $C$ et al. (2007) The different roles of cyclinD1-CDK4 in STP and mGluR-LTD during the postnatal development in mice hippocampus area CA1. BMC Dev Biol 7:57 doi: 10.1186/1471-213x-7-57

Li JM, Zhou J, Xu Z, Huang HJ, Chen MJ, Ji JS (2017) MicroRNA-27a-3p inhibits cell viability and migration through down-regulating DUSP16 in hepatocellular carcinoma. J Cell Biochem doi: $10.1002 / \mathrm{jcb} .26526$ 
Li L, Luo Z (2017) Dysregulated miR-27a-3p promotes nasopharyngeal carcinoma cell proliferation and migration by targeting Mapk10. Oncology Reports 37:2679-2687 doi: 10.3892/or.2017.5544

Liang J et al. (2017) miR-27a-3p targeting RXR $\alpha$ promotes colorectal cancer progression by activating Wnt/ß-catenin pathway. Oncotarget 8:82991-83008 doi: 10.18632/oncotarget.19635

Livak KJ, Schmittgen TD (2001) Analysis of relative gene expression data using real-time quantitative PCR and the 2(-Delta Delta C(T)) Method. Methods 25:402-408

Lord MS, Chuang CY, Melrose J, Davies MJ, lozzo RV, Whitelock JM (2014) The role of vascular-derived perlecan in modulating cell adhesion, proliferation and growth factor signaling. Matrix Biol 35:112-122 doi: 10.1016/j.matbio.2014.01.016

Nakamura R, Nakamura F, Fukunaga S (2015) Perlecan Diversely Regulates the Migration and Proliferation of Distinct Cell Types in vitro. Cells Tissues Organs 200:374-393 doi: 10.1159/000440950

Niu T-T, Zhang D-S, Chen H-M, Yan X-J (2015) Modulation of the binding of basic fibroblast growth factor and heparanase activity by purified $\lambda$-carrageenan oligosaccharides. Carbohydrate Polymers 125:76-84 doi: https://doi.org/10.1016/j.carbpol.2015.02.069

Nonaka R et al. (2015) Perlecan deficiency causes endothelial dysfunction by reducing the expression of endothelial nitric oxide synthase. Physiol Rep 3 doi: 10.14814/phy2.12272

Ohno S, Hirano S, Yasumoto A, Ikeda H, Takebayashi S, Miura M (2016) Outcome of regenerative therapy for age-related vocal fold atrophy with basic fibroblast growth factor. Laryngoscope 126:1844-1848 doi: 10.1002/lary.25578

Ong CT et al. (2007) Epithelial-mesenchymal interactions in keloid pathogenesis modulate vascular endothelial growth factor expression and secretion. J Pathol 211:95-108 doi: 10.1002/path.2081

Peng H, Wang X, Zhang P, Sun T, Ren X, Xia Z (2015) miR-27a promotes cell proliferation and metastasis in renal cell carcinoma. International Journal of Clinical and Experimental Pathology 8:2259-2266

Reinke JM, Sorg H (2012) Wound repair and regeneration. Eur Surg Res 49:35-43 doi: 10.1159/000339613

Sliwinska A, Kasinska MA, Drzewoski J (2017) MicroRNAs and metabolic disorders - where are we heading? Arch Med Sci 13:885-896 doi: 10.5114/aoms.2017.65229

Suarez E, Syed F, Alonso-Rasgado T, Bayat A (2015) Identification of biomarkers involved in differential profiling of hypertrophic and keloid scars versus normal skin. Arch Dermatol Res 307:115-133 doi: 10.1007/s00403-014-1512-4

Sun Y, Liu WZ, Liu T, Feng X, Yang N, Zhou HF (2015) Signaling pathway of MAPK/ERK in cell proliferation, differentiation, migration, senescence and apoptosis. J Recept Signal Transduct Res 35:600-604 doi: 10.3109/10799893.2015.1030412

Vedrenne N, Coulomb B, Danigo A, Bonte F, Desmouliere A (2012) The complex dialogue between (myo)fibroblasts and the extracellular matrix during skin repair processes and ageing. Pathol Biol (Paris) 60:20-27 doi: 10.1016/j.patbio.2011.10.002

Wang $X$ et al. (2017) Study on the role of Hsa-miR-31-5p in hypertrophic scar formation and the mechanism. Experimental Cell Research 361:201-209 doi: https://doi.org/10.1016/j.yexcr.2017.09.009

Warren CR, Kassir E, Spurlin J, Martinez J, Putnam NH, Farach-Carson MC (2015) Evolution of the perlecan/HSPG2 gene and its activation in regenerating Nematostella vectensis. PLoS One 
10:e0124578 doi: 10.1371/journal.pone.0124578

Webb AH et al. (2017) Inhibition of MMP-2 and MMP-9 decreases cellular migration, and angiogenesis in in vitro models of retinoblastoma. BMC Cancer 17:434 doi: 10.1186/s12885-017-3418-y

Wong R, Chen W, Zhong X, Rutka JT, Feng ZP, Sun HS (2018) Swelling-induced chloride current in glioblastoma proliferation, migration, and invasion. 233:363-370 doi: 10.1002/jcp.25891

$\mathrm{Xu} \mathrm{W}$ et al. (2013) miR-24-3p and miR-27a-3p promote cell proliferation in glioma cells via cooperative regulation of MXI1. Int J Oncol 42:757-766 doi: 10.3892/ijo.2012.1742

Yang H, Wu C, Xiao Y, Zhou S (2015) Connexin and fibrosis related microRNAs in complex fractionated atrial electrograms. Arch Med Sci 11:679-682 doi: 10.5114/aoms.2015.52375

Zhang $\mathrm{H}$ et al. (2010) Down-regulation of miR-27a might reverse multidrug resistance of esophageal squamous cell carcinoma. Dig Dis Sci 55:2545-2551 doi: 10.1007/s10620-009-1051-6

Zhou L et al. (2016) MiR-27a-3p functions as an oncogene in gastric cancer by targeting BTG2. Oncotarget 7:51943-51954 doi: 10.18632/oncotarget.10460

Zhu L, Wang Z, Fan Q, Wang R, Sun Y (2014) microRNA-27a functions as a tumor suppressor in esophageal squamous cell carcinoma by targeting KRAS. Oncol Rep 31:280-286 doi: 10.3892/or.2013.2807

Zhu Z, Ding J, Shankowsky HA, Tredget EE (2013) The molecular mechanism of hypertrophic scar. J Cell Commun Signal 7:239-252 doi: 10.1007/s12079-013-0195-5 


\section{Figure legends}

Figure 1. MicroRNA (miR)-27a-3p promotes proliferation and migration of NIH/3T3 cells. $\mathrm{NIH} / 3 \mathrm{~T} 3$ cells were transfected with miR-NC, miR-27a-3p mimic or miR-27a-3p inhibitor. Cells in the miR-NC group were served as control. A. Expression of miR-27a-3p by stem-loop RT-PCR. B. Cell viability by a Cell Counting Kit-8 assay. C. Expression of Cyclin D1 by Western blot analysis. D. Cell migration by Transwell assay. E. Expression of MMP-2 and MMP-9 by Western blot analysis. Data was presented as the mean \pm SD of at least three independent experiments. ${ }^{*} P<0.05 ;{ }^{*} P<0.01$ or ${ }^{* * *} P<0.001$ vs. miR-NC group. miR-NC, negative control of miR-27a-3p mimic and inhibitor; MMP, matrix metalloproteinase.

Figure 2. MicroRNA (miR)-27a-3p positively regulates perlecan expression in NIH/3T3 cells. A. NIH/3T3 cells were transfected with miR-NC, miR-27a-3p mimic or miR-27a-3p inhibitor. Cells in the miR-NC group were served as control. Protein expression of perlecan was determined by Western blot analysis. B. NIF/3T3 cells were co-transfected with pGL3-perlecan vector and increasing amounts of miR-27a-3p inhibitor, the relative luciferase activity was detected using dual luciferase reporter assay. Data was presented as the mean $\pm \mathrm{SD}$ of at least three independent experiments. ${ }^{*} P<0.05$; $* * P<0.01$ or $* * * P<0.001$ vs. miR-NC or $0 \mathrm{nM}$ miR-27a-3p inhibitor group. miR-NC, negative control of miR-27a-3p mimic and inhibitor.

Figure 3. MicroRNA (miR)-27a-3p affects NIH/3T3 cells via up-regulation of perlecan. A. Protein expression of perlecan in NIH/3T3 cells after si-perlecan transfection was detected by Western blot analysis. NIH/3T3 cells were co-transfected with miR-NC+si-NC, miR-27a-3pmimic + si-NC, si-perlecan + miR-NC or miR-27a-3p mimic + si-perlecan. B. Cell viability by a Cell Counting Kit-8 assay. C. Expression of Cyclin D1 by Western blot analysis. D. Cell migration by Transwell assay. E. Expression of MMP-2 and MMP-9 by Western blot analysis. Data was presented as the mean $\pm \mathrm{SD}$ of at least three independent experiments. ${ }^{*} P<$ $0.05 ; * * P<0.01$ or ${ }^{* * *} P<0.001$ vs. si-NC or miR-NC+si-NC group. ${ }^{\#} P<0.05$ or ${ }^{\# *} P<0.01$ vs. 
miR-27a-3p mimic + si-NC group. miR-NC, negative control of miR-27a-3p mimic and inhibitor; si-perlecan, small interfering RNA targeting perlecan; si-NC, negative control of si-perlecan; MMP, matrix metalloproteinase.

Figure 4. MicroRNA (miR)-27a-3p up-regulates expression levels of VEGF and bFGF via up-regulation of perlecan in $\mathrm{NIH} / 3 \mathrm{T3}$ cells. NIH/3T3 cells were co-transfected with miR-NC+si-NC, miR-27a-3p mimic + si-NC, si-perlecan+miR-NC or miR-27a-3p mimic + si-perlecan. Protein expression levels of VEGF and bFGF were determined by Western blot analysis. Data was presented as the mean $\pm \mathrm{SD}$ of at least three independent experiments. ${ }^{* *} P<0.01$ or ${ }^{* * *} P<0.001$ vs. miR-NC+si-NC group. ${ }^{\#} P<0.01$ or ${ }^{\#} P<0.001$ vs. miR-27a-3p mimic + si-NC group. miR-NC, negative control of miR-27a-3p mimic and inhibitor; si-perlecan, small interfering RNA targeting perlecan; si-NC, negative control of si-perlecan; VEGF, vascular endothelial growth factor; bFGF, basic fibroblast growth factor.

Figure 5. MicroRNA (miR)-27a-3p activates MAPK/ERK and PI3K/AKT pathways via up-regulation of perlecan in $\mathrm{NIH} / 3 \mathrm{T3}$ cells. $\mathrm{NIH} / 3 \mathrm{~T} 3$ cells were co-transfected with miR-NC+si-NC, miR-27a-3p mimic +si-NC, si-perlecan+miR-NC or miR-27a-3p mimic + si-perlecan. Expression levels of p-MAPK, t-MAPK, p-ERK and t-ERK (A) and p-PI3K, t-PI3K, p-AKT and t-AKT (B) WERE determined by Western blot analysis. Data was presented as the mean $\pm \mathrm{SD}$ of at least three independent experiments. ${ }^{* * *} P<0.001 \mathrm{vs}$. miR-NC+si-NC group. ${ }^{\# \# P} P<0.001$ vs. miR-27a-3p mimic+si-NC group. miR-NC, negative control of miR-27a-3p mimic and inhibitor; si-perlecan, small interfering RNA targeting perlecan; si-NC, negative control of si-perlecan; MAPK, mitogen-activated protein kinase; ERK, extracellular signal-regulated kinase; PI3K, phosphatidylinositol-3-kinase; p-, phospho-; t-, total. 
A

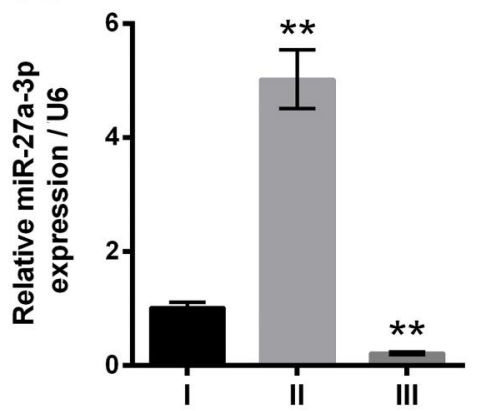

C

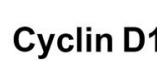

I II

Cyclin D1

$\beta$-actin

III

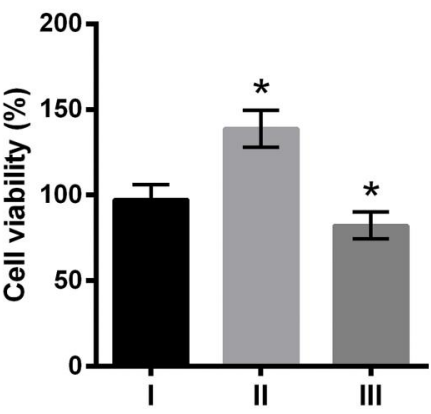

D



E

MMP-2

$34 \mathrm{KDa}$

MMP-9

$42 \mathrm{KDa}$
$74 \mathrm{KDa}$

$92 \mathrm{KDa}$

$42 \mathrm{KDa}$

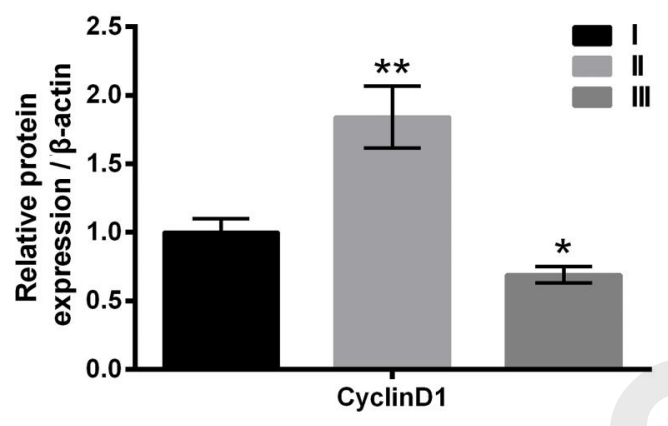

I: miR-NC

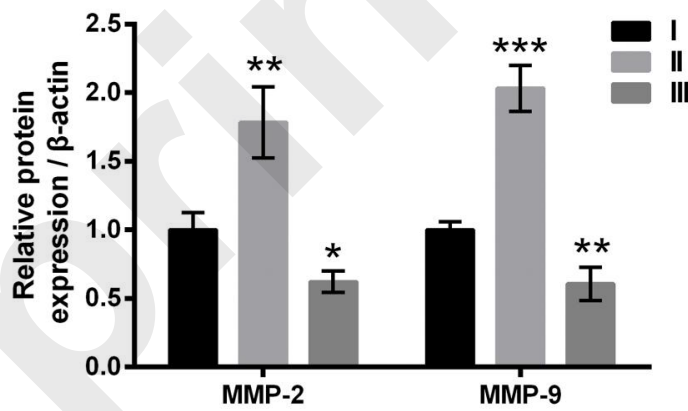

III: miR-27a-3p inhibitor 


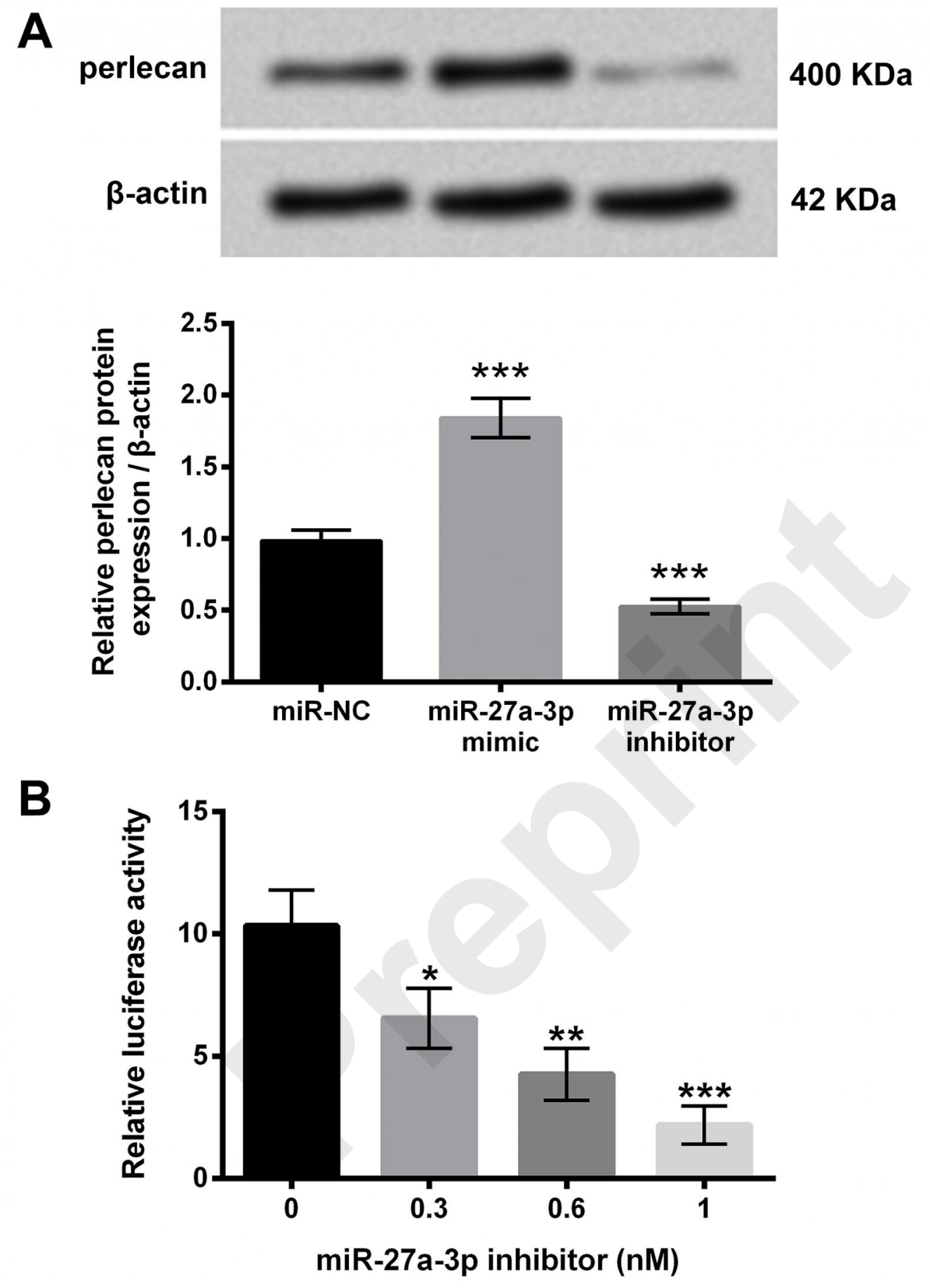


A

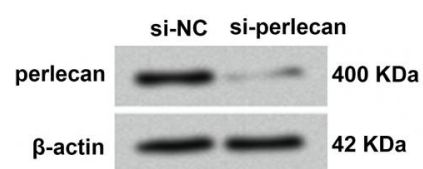

C

E
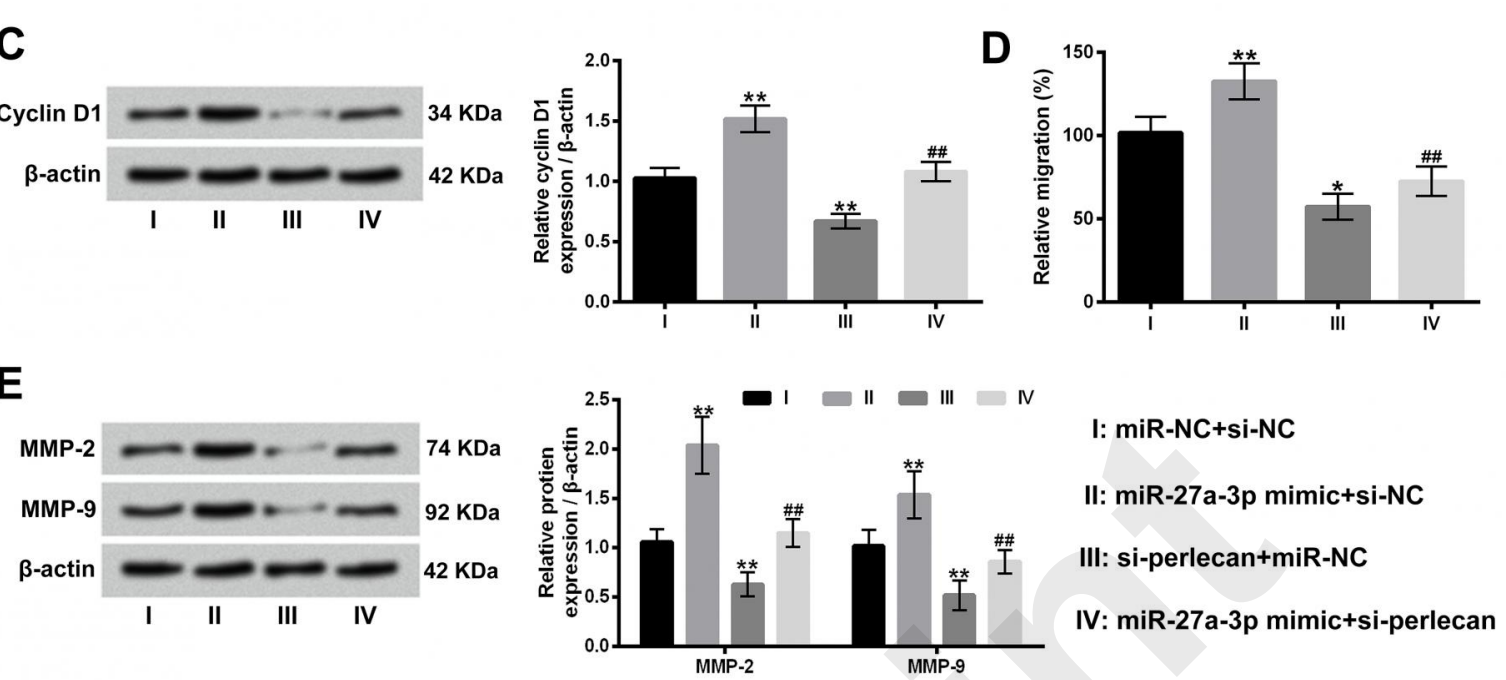

B

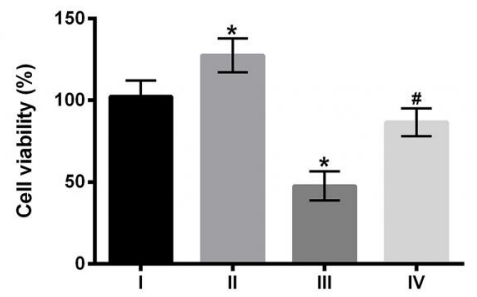



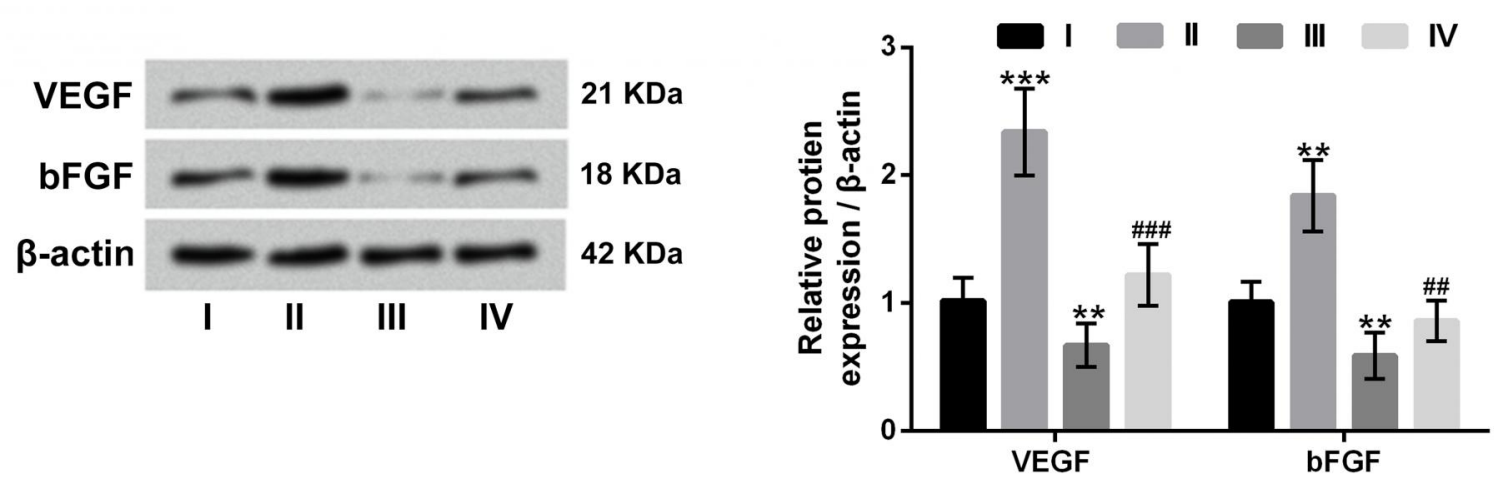

I: miR-NC+si-NC II: miR-27a-3p mimic+si-NC III: si-perlecan+miR-NC IV: miR-27a-3p mimic+si-perlecan 



I: miR-NC+si-NC

II: miR-27a-3p mimic+si-NC

III: si-perlecan+miR-NC

IV: miR-27a-3p mimic+si-perlecan 
Figure 1C

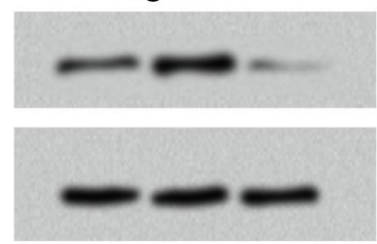

Figure 4

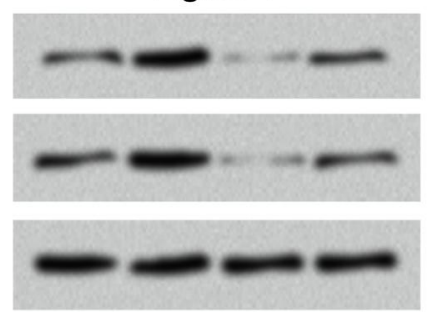

Figure 3A



Figure 1E
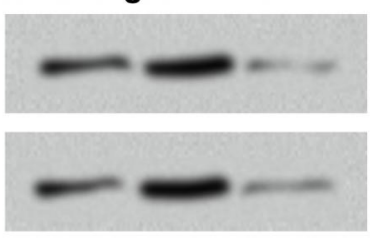

$-0$

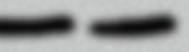

Figure 3C

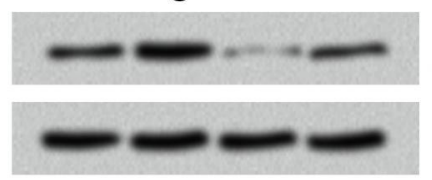

Figure 5A

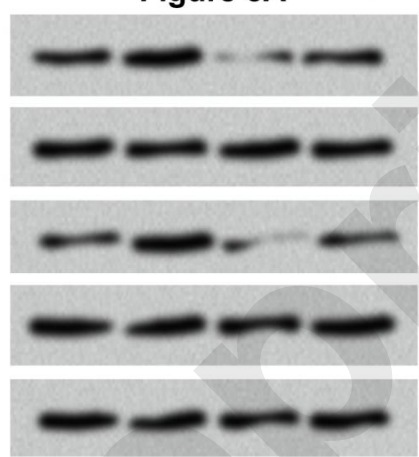

Figure 2A

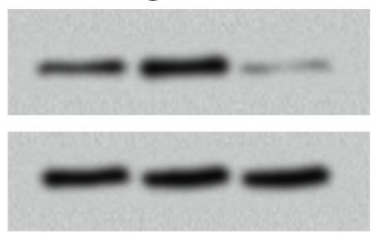

Figure 3E

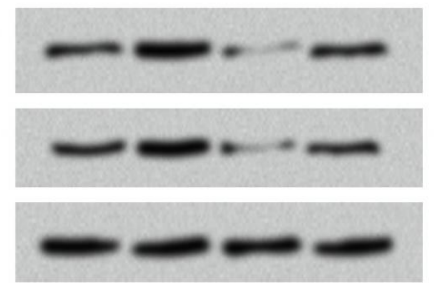

Figure 5B

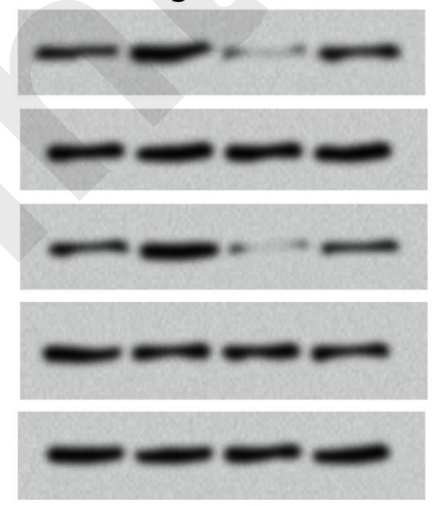

